\title{
Time-Dependent Solution for a Star Immersed in a Background Radiation
}

\author{
Hongya Liu* and Qiuyang Zhang \\ Department of Physics, Dalian University of Technology, \\ Dalian, 116024, P.R. China
}

\begin{abstract}
We study a time-dependent and spherically-symmetric solution with a star-like source. We show that this solution can be interpreted as an exterior solution of a contracting star which has a decreasing temperature and is immersed in a homogenous and isotropic background radiation. Distribution of the temperature in the fields and close-to Schwarzschild approximation of the solution are studied. By identifying the radiation with the cosmic background one, we find that the close-to-Schwarzschild approximate solution is valid in a wide range in our solar system. Possible experimental tests of the solution are discussed briefly.
\end{abstract}

PACS number(s): 04.20.-q, 04.50.+h

*Email: hyliu@dlut.edu.cn 


\section{INTRODUCTION}

It is well known that spherically-symmetric sources such as stars can be modelled in the simplest way by the interior and exterior Schwarzschild solutions. But to include the radiation outside a star, more complicated solutions are required. These include the Vaidya metric which uses a retarded time coordinate to describe a radiating atmosphere, matter are matched to exterior spacetimes, which extend the Vaidya solution to include both a radiation field and a string fluid. Recently, Liu and Wesson presented a new kind of solutions in which the metric is time-dependent (but not of the Vaidya form) and the energy-momentum tensor is of the form of a perfect fluid for radiation plus a radial heat flow. Clearly, this solution describes sources which are time-dependent and spherically symmetric. However, we wish to know specifically what kind of sources the solution represents. In this paper we will show that it can describe exterior fields of a contracting (or expanding) star immersed in a homogenous and isotropic background radiation.

\section{4D SOLUTION DERIVED FROM 5D SOLUTIONS}

Campbell's theorem says that any 4D Einstein solution with a source can be locally embedded in a 5D manifold without sources whose field equations in terms of the Ricci tensor are $R_{A B}=0.0$ (Here and elsewhere lower case Greek letter run 0,123 and uppercase Latin letters run $0,123,4$, and we use units $c=1$.) A major application of Campbell's theorem is to study embeddings of known 4D Einstein solutions in 5D Ricci flat manifolds. Another application of the theorem is to generate new 4D solutions from known 5D Ricci flat solutions.7,10 In the following we will show briefly how a new 4D solution is generated in Ref.[7]. For the purpose of convenience, some of the equations and notations in Ref. [7] will be re-expressed.

In Reference [7], Liu and Wesson presented a class of 5D solutions. This class of solutions is time-dependent in 5D and spherically symmetric in $3 \mathrm{D}$ with the $5 \mathrm{D}$ metric being

$$
d S^{2}=B(r) d t^{2}-(1-\lambda t)^{2}\left[A(r) d r^{2}+r^{2}\left(d \theta^{2}+\sin ^{2} \theta d \varphi^{2}\right)\right]-(1-\lambda t)^{-4} d y^{2},
$$

where $\lambda$ is a constant and the two functions $B$ and $A$ are determined by

$$
\begin{aligned}
& \frac{B^{\prime}}{B}=3 \lambda^{2} r \frac{A}{B}+\frac{A-1}{r}, \\
& \frac{A^{\prime}}{A}=3 \lambda^{2} r \frac{A}{B}-\frac{A-1}{r},
\end{aligned}
$$

where a prime denotes derivative with respect to $r$. Clearly, (2) and (3) can be solved, at least numerically, by imposing boundary conditions on $B(r)$ and $A(r)$. This $5 \mathrm{D}$ 
solution satisfies the $5 \mathrm{D}$ equations $R_{A B}=0$ and therefore is $5 \mathrm{D}$ empty. However, the $4 \mathrm{D}$ part of the $5 \mathrm{D}$ metric (1), together with the two equations (2) and (3), defines a 4D solution as shown in the following:

$$
\begin{gathered}
d s^{2}=B(r) d t^{2}-(1-\lambda t)^{2}\left[A(r) d r^{2}+r^{2}\left(d \theta^{2}+\sin ^{2} \theta d \varphi^{2}\right)\right], \\
\frac{B^{\prime}}{B}=3 \lambda^{2} r \frac{A}{B}+\frac{A-1}{r}, \\
\frac{A^{\prime}}{A}=3 \lambda^{2} r \frac{A}{B}-\frac{A-1}{r}, \\
8 \pi G T_{\alpha \beta} \equiv G_{\alpha \beta},
\end{gathered}
$$

where $T_{\alpha \beta}$ is an effective or induced energy-momentum tensor with

$$
\begin{aligned}
8 \pi G T_{0}^{0} & =\frac{6 \lambda^{2}}{B(1-\lambda t)^{2}}, \\
8 \pi G T_{1}^{1} & =8 \pi G T_{2}^{2}=8 \pi G T_{3}^{3}=-\frac{2 \lambda^{2}}{B(1-\lambda t)^{2}}, \\
8 \pi G T_{0}^{1} & =\frac{\lambda B^{\prime}}{A B(1-\lambda t)^{3}} .
\end{aligned}
$$

It was also shown in Ref.[7] that this $T_{\alpha \beta}$ can be modeled as a perfect fluid plus a radial heat flow,

$$
T_{\alpha \beta}=(\rho+p) u_{\alpha} u_{\beta}-p g_{\alpha \beta}+q_{\alpha} u_{\beta}+u_{\alpha} q_{\beta}
$$

were $\rho$ is the mass density, $p$ is the pressure, $u_{\alpha}=\left(u^{0}, 0,0,0\right)$ is the 4 -velocity, $q_{\alpha}=$ $\left(0, q^{1}, 0,0\right)$ is the heat-flux vector, and $u_{\alpha}$ and $q_{\alpha}$ obey the orthogonality condition $q_{\alpha} u^{\alpha}=0$. Then equations (8)-([10) yield

$$
\begin{aligned}
\rho & =3 p=\frac{3 \lambda^{2}}{4 \pi G B(1-\lambda t)^{2}}, \\
q^{1} & =\frac{\lambda B^{\prime}}{8 \pi G A B^{3 / 2}(1-\lambda t)^{3}} .
\end{aligned}
$$

Equations (4)-(13) constitute a complete set of the 4D solution, from which we see that the equation of state of the $4 \mathrm{D}$ fluid is $\rho=3 p$, so it represents a radiation or extra-relativistic particles accompanied by a radial heat flow.

\section{HEAT FLOW AND TEMPERATURE OF THE FIELDS}

The induced 4D energy-momentum tensor (11) describes a thermodynamical system in which the radial heat current $q_{\alpha}$ implies a radial temperature gradient. Now 
we wish to calculate this temperature distribution over the fields. The generalized relativistic relation between heat current and temperature gradient can be found in Refs.[11,12] with

$$
q^{\alpha}=-\kappa\left(T,_{\mu}-\dot{u}_{\mu} T\right) h^{\alpha \mu}, \quad \dot{u}_{\mu} \equiv u_{\mu ; \nu} u^{\nu},
$$

where $\kappa$ is the coefficient of the thermal conductivity and $h^{\alpha \mu}$ is the projection tensor,

$$
h^{\alpha \beta}=u^{\alpha} u^{\beta}-g^{\alpha \beta} .
$$

To calculate equations (14), we calculate $\dot{u}_{\mu}$ firstly. With use of (田), we find the only non-vanishing $\dot{u}_{\mu}$ being $\dot{u}_{1}=-B^{\prime} /(2 B)$. Furthermore, we assume $T=T(t, r)$. Then equations (14) reduce to $q^{0}=q^{2}=q^{3}=0$ and

$$
q^{1}=-\frac{\kappa}{A(1-\lambda t)^{2}}\left(T,,_{1}+\frac{B^{\prime}}{2 B} T\right) .
$$

Combining (13) and (16), we get

$$
-\kappa\left(T,_{1}+\frac{B^{\prime}}{2 B} T\right)=\frac{\lambda B^{\prime}}{8 \pi G(1-\lambda t) B^{3 / 2}} .
$$

We find that this equation can be integrated, giving an exact solution being

$$
\sqrt{B(r)} T(t, r)=\sqrt{B_{R}} T_{R}(t)+\frac{\lambda}{8 \pi G \kappa(1-\lambda t)} \ln \frac{B_{R}}{B(r)},
$$

where $R$ is a constant radius, and $B_{R}$ and $T_{R}(t)$ are values of $B(r)$ and $T(t, r)$ at $r=R$, respectively. From (13) we see that $\lambda=0$ corresponds to thermal equilibrium

$q^{\alpha}=0$. Then, from (18), if $\lambda=0$ we get $\sqrt{B} T=$ constant. Thus we recover the conclusion that thermal equilibrium corresponds not to constant temperature, but to the redshifted temperature distribution $\sqrt{g_{00}} T=$ constant 11 Generally we have $\lambda \neq 0$ and the exact solution (18) determines both the space distribution and the time variation of the temperature $T$ over the fields.

\section{CLOSE-TO-SCHWARZSCHILD APPROXIMATION}

The close-to-Schwarzschild approximation of the solution (4) was given in Ref.[7]. We find that it can be re-expressed in the following form:

$$
\begin{aligned}
d s^{2} & =B(r) d t^{2}-(1-\lambda t)^{2}\left[A(r) d r^{2}+r^{2}\left(d \theta^{2}+\sin ^{2} \theta d \varphi^{2}\right)\right], \\
B & =1-\frac{2 G M}{r}+2 \lambda^{2} r^{2}+O\left(\varepsilon^{3}\right), \\
A^{-1} & =1-\frac{2 G M}{r}-\lambda^{2} r^{2}+O\left(\varepsilon^{3}\right) .
\end{aligned}
$$


Here $\varepsilon$ is a small quantity of the order of the Newtonian potential $G M / r, O\left(\varepsilon^{3}\right)$ are terms of the order of $\varepsilon^{3}$ or higher, and we have assumed, for practical usage, that $\lambda r$ is also a small quantity of the order $\varepsilon$, i.e.,

$$
|\lambda| r \sim G M / r \sim \varepsilon \ll 1
$$

To verify the accuracy of this solution, one can calculate $B^{\prime} / B$ and $A^{\prime} / A$ firstly, and then substitute them into the two equations (2) and (3). Thus the approximate solutions (19)-(21) are correct in the range (22), or, equivalently, in

$$
G M \ll r \ll|\lambda|^{-1}
$$

We also conclude that (19)-(21) are accurate up to the second order of $\varepsilon$ and give back to the Schwarzschild solution if $\lambda=0$. So generally we can interpret the solution as an exterior solution of a star-like source. We will show, in the next section, that in a wide range in our solar system the condition (22), or, equivalently, (23), is satisfied.

Consider now the equation (12), which, by (20), reduces to

$$
\rho=3 p=\frac{3 \lambda^{2}}{4 \pi G(1-\lambda t)^{2}}\left[1+\frac{2 G M}{r}+O\left(\varepsilon^{2}\right)\right] .
$$

Here $\rho$ and $p$ constitute a perfect fluid with the equation of state $\rho=3 p$, implying a property for radiation or ultra-relativistic particles. From (24) we see that neglecting higher-order terms in the square bracket in the RHS of (24), the densities $\rho$ and $p$ are homogenous and isotropic. Thus we find that one can not interpret the fluid as a radiating atmosphere of a star such as in the Vaidya metric. interpret it as to describe a star immersed in a homogenous and isotropic background radiation.

The existence of the heat flow term in the energy-momentum tensor (11) implies that there must be a temperature gradient in the field and a heat interchanges between the star and the background radiation. Using (20) and (21) in (18) gives

$$
\sqrt{B(r)} T(t, r)-\sqrt{B_{R}} T_{R}(t)=\frac{\lambda M}{4 \pi \kappa(1-\lambda t)}\left(\frac{1}{r}-\frac{1}{R}\right)[1+O(\varepsilon)] .
$$

If we choose $R$ as the radius of the star, then in the exterior $r>R$, equation (25) implies that if $\lambda>0$ then $\sqrt{B(r)} T(t, r)<\sqrt{B_{R}} T_{R}(t)$. Be aware that the thermal equilibrium corresponds to $\sqrt{B(r)} T(t, r)=\sqrt{B_{R}} T_{R}(t)$ for which there is no heat flow. 11 So we conclude that the heat flows outwards if $\lambda>0$ and inwards if $\lambda<0$. This agree with the original relation (13) in which $q^{1}>0$ for $\lambda>0$ and $q^{1}<0$ for $\lambda<0$ since $B^{\prime}>0$ according to (20). Meanwhile, from the metric (19) and the result (24) we also see that if $\lambda>0$, then as the time $t$ increases, the 3D space contracts and the energy density of the outside fluid increases. All these properties are physically reasonable. 


\section{DISCUSSION}

As we have concluded in section 4 that the $4 \mathrm{D}$ solution discussed in this paper can be interpreted as an exterior solution of a spherical source such as a star which has a non-zero temperature and is immersed in a homogenous and isotropic background radiation. A natural candidate for this kind of radiation is the cosmic background radiation, for which the temperature is about $T_{0} \approx 2.7 \mathrm{~K}$ at present days with an energy density around $\rho_{b} \approx 4.0 \times 10^{-13} \mathrm{erg}_{\mathrm{cm}}^{-3}$. Thus, by using (12), we determine the constant $\lambda$ as

$$
|\lambda|^{-1} \approx \sqrt{\frac{3 c^{4}}{4 \pi G \rho_{b}}} \approx 2.7 \times 10^{30} \mathrm{~cm} \approx 1.8 \times 10^{17} \mathrm{AU} .
$$

So $\lambda$ is of the order of the Hubble constant. Now we wish to know if the close-toSchwarzschild solution given in (19)-(21) can describe our solar system. That is, we need to calculate and compare orders of the two terms $G M / r$ and $\lambda^{2} r^{2}$ appeared in (20) and (21). The average distance between the Sun and the nearest planet Mercury is about $0.387 \mathrm{AU}$. So we have

$$
\frac{G M_{\odot}}{R_{M e r c} c^{2}} \approx 2.55 \times 10^{-8}, \quad|\lambda| R_{M e r c} \approx 2 \times 10^{-18}
$$

The average distance between the Sun and the Pluto is about 39.53 AU. So

$$
\frac{G M_{\odot}}{R_{\text {Pluto }} c^{2}} \approx 2.5 \times 10^{-10}, \quad|\lambda| R_{\text {Pluto }} \approx 2 \times 10^{-16}
$$

Therefore we conclude that in a wide range in our solar system, we have $|\lambda| r \ll$ $G M / r$. So $\lambda^{2} r^{2}$ is of the order higher than the post-Newtonian order. This implies that the contributions of the cosmic background radiation to all of the known solar system experiments 13 are negligible. However, there may have other ways to detect possible new effects of the solution. For example, according to the time-dependent metric (19) and the value of $\lambda$ in (26), the radius of the central star should be contracting or expanding, depending on whether $\lambda$ is positive or negative, with a relative rate at present time being

$$
\left(\frac{\dot{R}}{R}\right)_{0}=-\lambda c \approx-3.5 \times 10^{-13} \text { yer }^{-1}, \quad \text { for } \lambda>0 \text {. }
$$

It is worth to study whether this kind of effect is observable.

\section{ACKNOWLEDGMENTS}

We thank Paul Wesson, Mark Roberts and Guowen Peng for comments. This work was supported by NSF of P. R. China under grant 19975007. 


\section{Research Bibliography}

${ }^{1}$ D. Kramer, H. Stephani, M. MacCallum, E. Herlt, Exact Solutions of Einstein's Field Equations, Cambridge Un. Press, Cambridge (1980).

${ }^{2}$ P.C. Vaidya, Nature (London) 171, 260 (1953).

${ }^{3}$ L. Herrera, J. Jimenez, L. Leal, J. Ponce de Leon, M. Esculpi, V. Galina, J. Math. Phys. 25, 3274 (1984).

${ }^{4}$ L. Herrera, J. Ponce de Leon, J. Math. Phys. 26, 2302 (1985); 26, 2847 (1985).

${ }^{5}$ M. Esculpi, L. Herrera, Phy. Rev. D 45, 3341 (1992).

${ }^{6}$ E. Glass, J.P. Krisch, Phys. Rev. D 57, R5945 (1998), gr-qc/9803040; Class. Quant. Grav. 16, 1175 (1999), gr-qc/9901034; Gen. Rel. Grav. 33, 1449 (2001), grqc/0103077.

${ }^{7}$ H. Liu and P.S. Wesson, J. Math. Phys. 42, 4963 (2001), gr-qc/0104009.

${ }^{8}$ J.E. Campbell, A Course of Differential Geometry, Clarendon, Oxford (1926); C. Romero, R. Tavakol, and R. Zalaletdinov, Gen. Rel. Grav. 28, 365 (1996).

${ }^{9}$ P.S. Wesson, Space, Time, Matter, World Scientific, Singapore (1999); J.M. Overduin and P.S. Wesson, Phys. Rep.283, 303 (1997), gr-qc/9805018; J. Ponce de Leon, Gen. Rel. Grav. 20, 539 (1988); H. Liu, B. Mashhoon, Ann. Phys. (Leipzig) 4, 565 (1995); J.E. Lidsey, C. Romero, R. Tavakol, and S. Rippl, Class. Quant. Grav. 14, 865 (1997), gr-qc/9907040; H. Liu and P.S. Wesson, Astrophys. J. 562, 1 (2001), gr-qc/0107093; S.S. Seahra and P.S. Wesson, Class. Quant. Grav. 19, 1139 (2002), Er-qc/0202010.

${ }^{10}$ H. Liu and P.S. Wesson, J. Math. Phys. 33, 3888 (1992).

${ }^{11}$ See, for example, C.W. Misner, K.S. Thorne, and J.A. Wheeler, Gravitation, Freeman, San Francisco (1973), p567.

${ }^{12}$ H. Stephani, General Relativity, Cambridge University Press, Cambridge (1982).

${ }^{13}$ C.M. Will, Theory and Experiment in Gravitational Physics, Cambridge University Press, Cambridge (1993). 